\title{
Wide-Field Spectral Super-Resolution Mapping of Optically Active Defects in Hexagonal Boron Nitride
}

\author{
Jean Comtet, ${ }^{* \dagger}{ }^{\dagger}$ Evgenii Glushkov, ${ }^{\dagger}$ Vytautas Navikas, ${ }^{\dagger}$ Jiandong Feng, ${ }^{*}, \star$ Vitaliy Babenko, ${ }^{\S}$ \\ Stephan Hofmann, ${ }^{, \odot}$ Kenji Watanabe,, Takashi Taniguchi," and Aleksandra Radenovic*, ${ }^{+} \odot$
}

${ }^{\dagger}$ Laboratory of Nanoscale Biology, Institute of Bioengineering, School of Engineering, École Polytechnique Fédérale de Lausanne (EPFL), 1015 Lausanne, Switzerland

${ }^{\ddagger}$ Laboratory of Experimental Physical Biology, Department of Chemistry, Zhejiang University, Hangzhou, 310027, China

${ }^{\S}$ Department of Engineering, University of Cambridge, JJ Thomson Avenue, CB3 0FA Cambridge, United Kingdom

"National Institute for Materials Science, 1-1 Namiki, Tsukuba 306-0044, Japan

Supporting Information

\begin{abstract}
Point defects can have significant impact on the mechanical, electronic, and optical properties of materials. The development of robust, multidimensional, high-throughput, and large-scale characterization techniques of defects is thus crucial for the establishment of integrated nanophotonic technologies and material growth optimization. Here, we demonstrate the potential of wide-field spectral single-molecule localization microscopy (SMLM) for the determination of ensemble spectral properties as well as the characterization of spatial, spectral, and temporal dynamics of single defects in chemical vapor deposition (CVD)-grown and irradiated

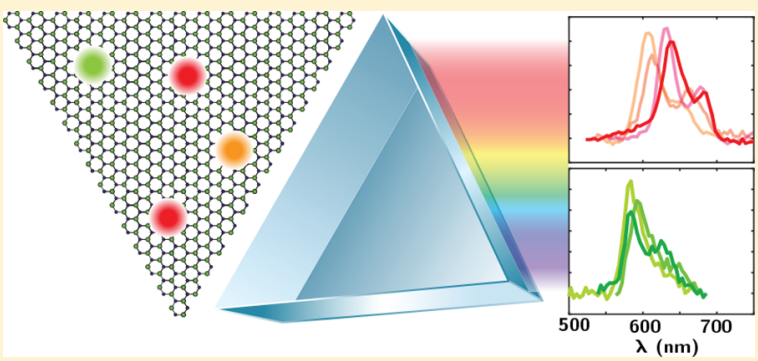
exfoliated hexagonal boron-nitride materials. We characterize the heterogeneous spectral response of our samples and identify at least two types of defects in CVD-grown materials, while irradiated exfoliated flakes show predominantly only one type of defects. We analyze the blinking kinetics and spectral emission for each type of defects and discuss their implications with respect to the observed spectral heterogeneity of our samples. Our study shows the potential of wide-field spectral SMLM techniques in material science and paves the way toward the quantitative multidimensional mapping of defect properties.
\end{abstract}

KEYWORDS: Super-resolution microscopy, spectral characterization, hexagonal boron nitride, 2D materials, defects

$\mathrm{D}$ efects in wide band gap semiconductor materials lead to the creation of energy states well within the band gap, conferring these materials with new and exciting properties due to quantum confinement. A popular and well-studied example of point defects are nitrogen-vacancy (NV) centers in diamond, which can act as single-photon emitters, ${ }^{1}$ ultrasensitive magnetometers, ${ }^{2}$ and biological sensors. ${ }^{3}$

In recent years, 2D semiconductors and insulators have emerged as new classes of materials able to host functional defects. Single-photon emission from such defects has been demonstrated in 2D transition-metal dichalcogenides at cryogenic temperatures $^{4-7}$ and in hexagonal boron nitride $(\mathrm{hBN})$ at room temperature. ${ }^{8}$ Defects in $2 \mathrm{D}$ materials show great promise for many applications in integrated photonics. ${ }^{9,10}$ The $2 \mathrm{D}$ nature of the material favors integration in photonic circuits while allowing high light-extraction efficiency. ${ }^{10}$ Defects in $2 \mathrm{D}$ materials also exhibit high sensitivity to their environment, leading to tunable properties ${ }^{11}$ and allowing deterministic positioning of emitters through strain engineering. ${ }^{12-14}$

However, defects can also be detrimental, leading to a decrease in the electrical and mechanical properties of materials. For example, the use of $\mathrm{hBN}$ as an encapsulation layer in nanoelectronics ${ }^{15}$ can be dramatically affected by the presence of defects, causing increased scattering or facilitated dielectric breakdown ${ }^{16}$ and precluding, for now, the use of CVD-grown hBN materials as efficient insulating layers in nanoelectronics. ${ }^{17}$

Due to their atomic-scale nature, characterizing defects can be very difficult. Defects can be imaged with high spatial resolution in a transmission electron microscope (TEM) ${ }^{18}$ but TEM imaging by itself tends to induce more defects in the sample and is restricted to very small $\left(\sim 10 \mathrm{~nm}^{2}\right)$ areas. Highresolution scanning probe microscopy techniques have allowed the characterization of $2 \mathrm{D}$ materials at the single-defect level, ${ }^{19}$ and 2D material systems have also been investigated using near-field scanning optical microscopy (NSOM). ${ }^{20}$ Unfortunately, all of these scanning techniques share similar limitations as TEM concerning time-consuming sample preparation and small imaging areas. Finally, confocal techniques ${ }^{8}$ have been successful at characterizing the optical properties of single defects but require very sparse samples due to diffraction-

Received: January 15, 2019

Revised: February 24, 2019

Published: March 13, 2019 
a

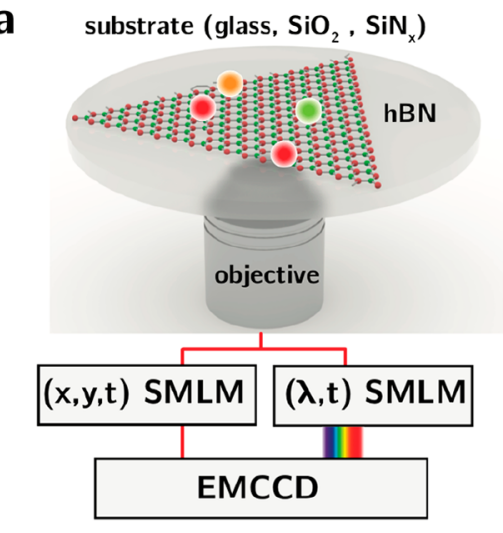

b

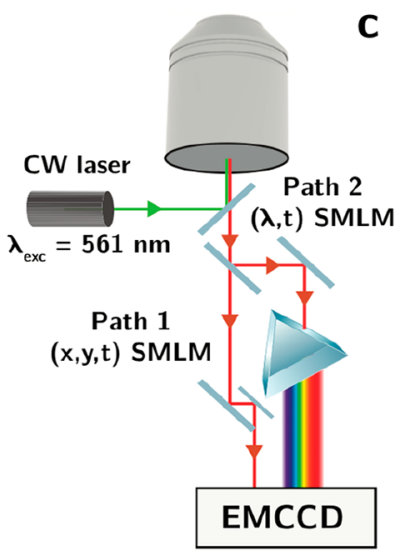

C

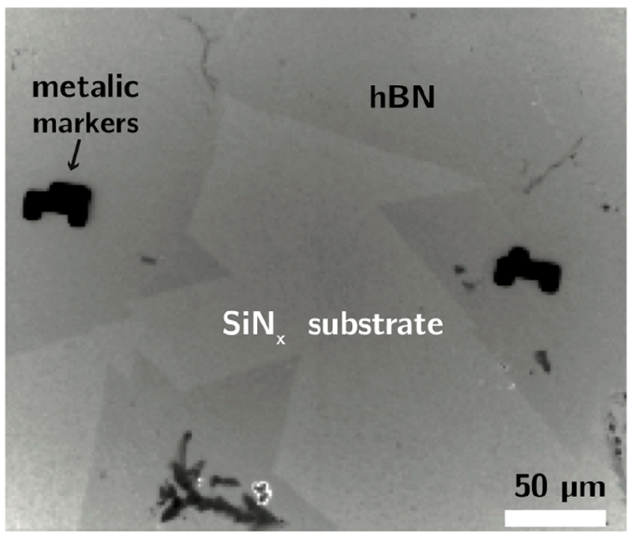

d
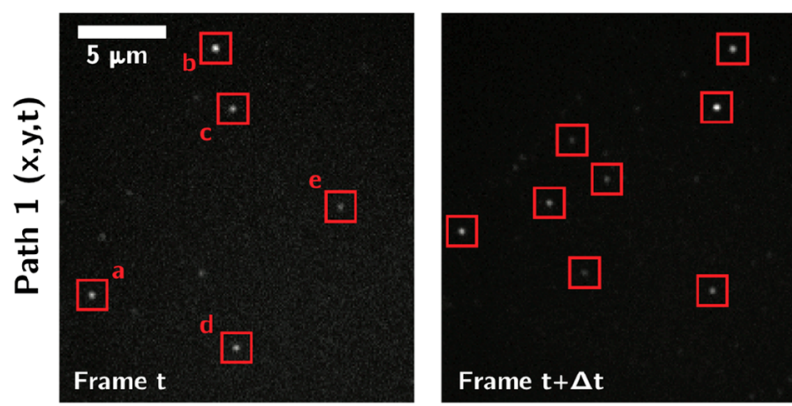

e
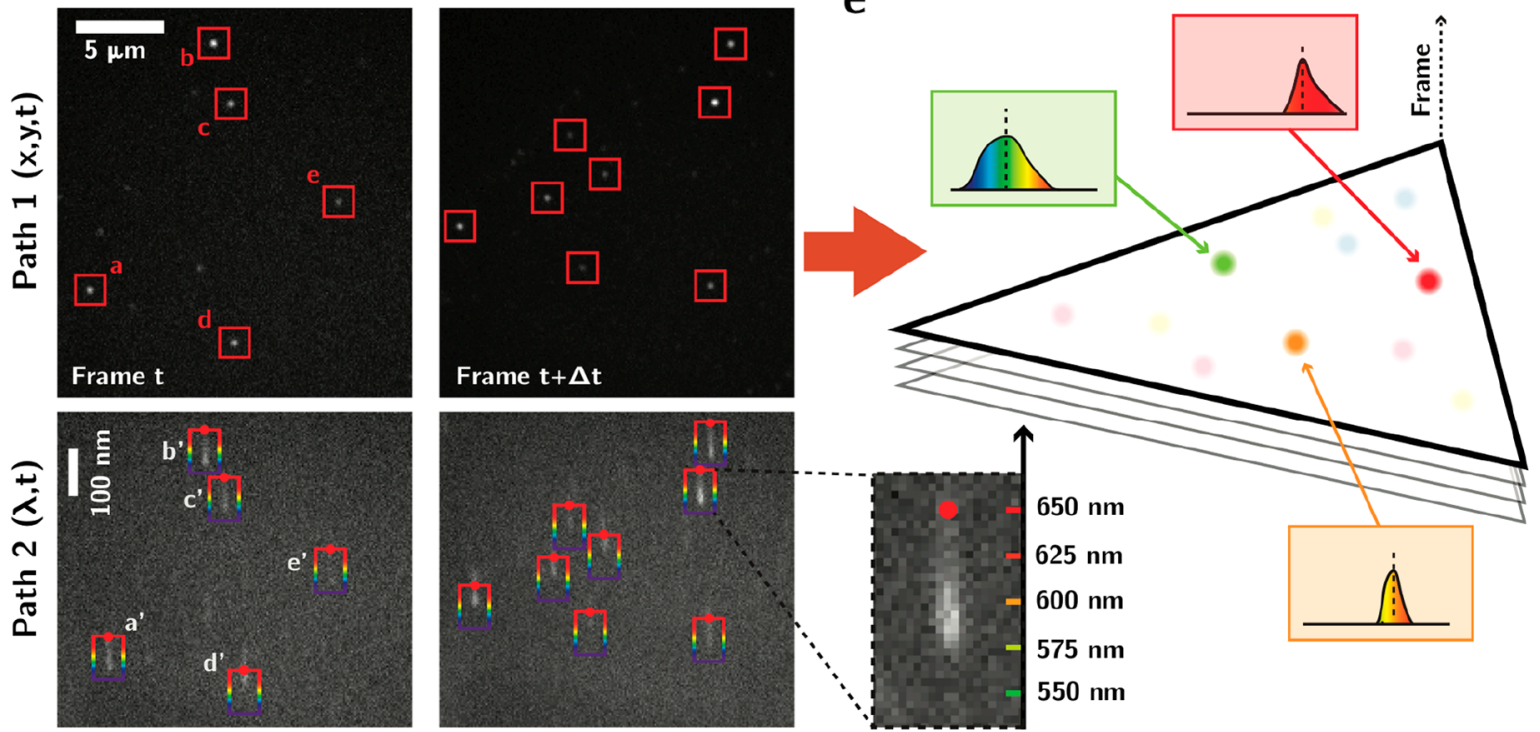

Figure 1. Ultrahigh-throughput prism-based wide field spectral characterization of optical emitters in hexagonal boron nitride (hBN). (a) Principle of the experimental setup, allowing the spatial and spectral single-molecule localization microscopy (SMLM) of emitters in hBN materials deposited on various substrates (glass, $\mathrm{SiO}_{2}$, and $\mathrm{SiN}_{x}$ chips). EMCCD: electron multiplying charge-coupled device. (b) Schematic of the experimental setup. Upon laser excitation, fluorescence signal emanating from single defects is collected by a high-NA objective and split toward two distinct paths for spatial (path 1) and spectral (path 2) characterization. Spatial path 1 leads to diffraction-limited spots for individual emitters, which can be localized with subpixel accuracy. Spectral path 2 is composed of a dispersive prism element, shifting the fluorescence of individual emitters according to their emission wavelength. Images from both paths are then projected on the same chip of an EMCCD camera (see panel d). (c) Wide field image of CVD grown $\mathrm{hBN}$ flakes transferred on $\mathrm{Si} / \mathrm{SiN}_{x}$ chips. (d) Simultaneously recorded wide field image (path $\left.1 ; x, y, t\right)$ and spectral image (path $2 ; \lambda, t)$ of emitters in exfoliated hBN flakes between successive frames at $t$ and $t+\Delta t$. The red boxes in Path 1 indicate spatial position of individual emitters, corresponding to diffraction-limited spots, localized with sub-pixel accuracy. The multicolored boxes in path 2 show the corresponding images of individual emitters after vertical dispersion by the prism element. The red dots in spectral channel indicate the mapped spectral position of $650 \mathrm{~nm}$ for each emitter in the spatial channel. The one-to-one correspondence between spatial and spectral path allows us to obtain the full spectra of each individual emitters through the measurement of the vertical shift in the spectral channel (see the zoomed-in section of panel d). Images are averaged over 5 frames for clarity (with $20 \mathrm{~ms}$ exposure). (e) Principle of the reconstructed spectral super-resolved image. Spatial positions of emitters, along with their attributed spectra, are summed up over successive frames, allowing the reconstruction of a superresolved map.

limited imaging. There is, thus, a clear demand in the development of new strategies that allow the large-area characterization of defects in 2D materials.

With this goal in mind, we recently established singlemolecule localization microscopy (SMLM) as a viable strategy for the wide-field mapping of optically active defects in hBN. ${ }^{18}$ Using the transient emission properties of individual emitters, we could separate emitters spatially down to $10 \mathrm{~nm} .^{18}$ However, purely spatial SMLM techniques are still hampered by their lack of contrast, which can lead to the substantial overcounting or under-counting of defect's densities and precludes the multidimensional measurement of defect's properties, such as polarization, and spectra.

In this work, we focus on prototypical optically active defects in $\mathrm{hBN}$ and demonstrate that the implementation of spectral information concomitant to spatial SMLM (obtained by placing a prism in the detection path) is a valuable step forward in the large-area, nondestructive characterization of 2D materials and beyond. Our combined wide-field spectral and spatial super-resolved technique allows the determination of statistical ensemble spectral properties as well as the extraction of spatial, spectral and temporal dynamics of single defects. We demonstrate our approach on both monolayers of CVD-grown 
a

C
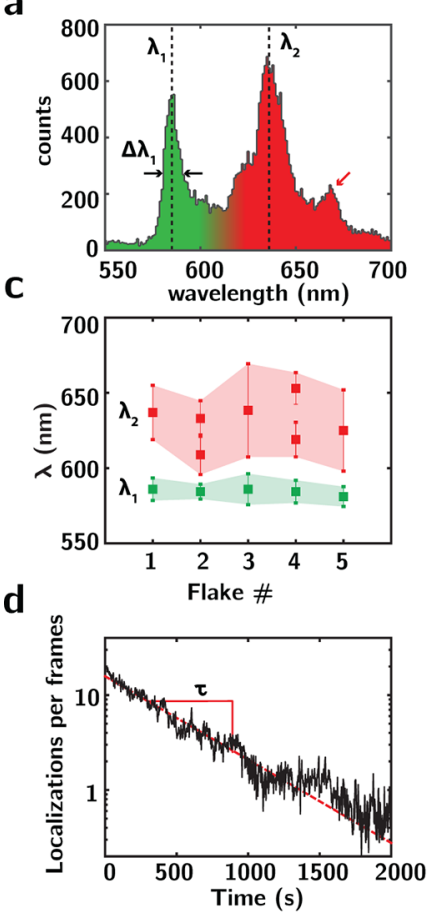

b

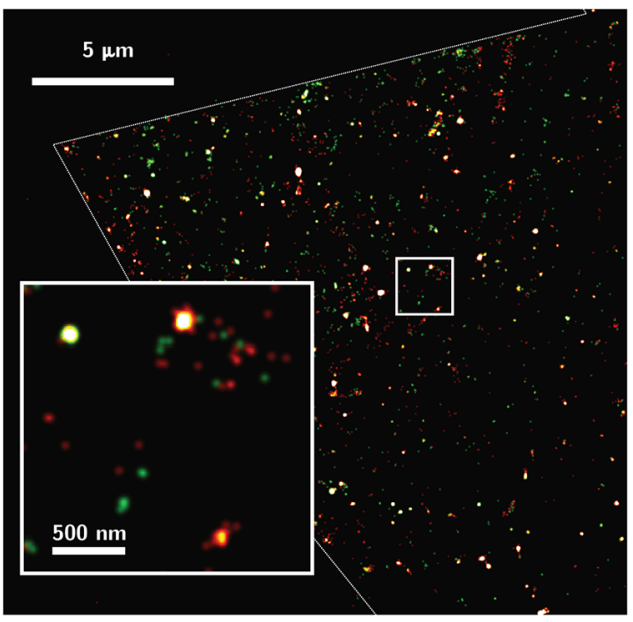

e

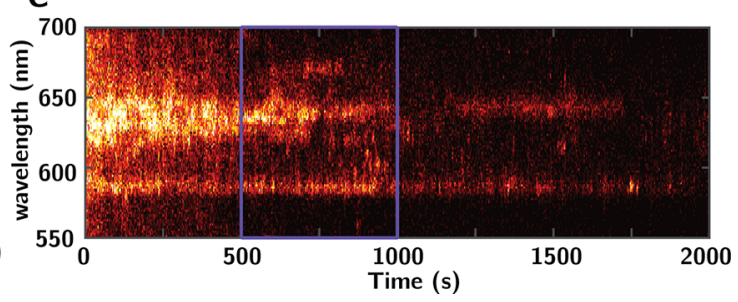

Figure 2. Wide-field super-resolved spectral and spatial map of single emitters in CVD-grown hBN flakes. (a) The spectral distribution of center emission wavelengths for a CVD-grown hBN flake. A dual distribution is observed, corresponding to green and red emitters of respective center emission wavelength $\lambda_{1}$ and $\lambda_{2}$. The smaller emission peak, indicated by the red arrow, corresponds to a phonon sideband (see the text for details). The bin size is $1 \mathrm{~nm}$. (b) Corresponding spatial map with spectral contrast, showing the position of the two types of emitters throughout the flake. Localization events are rendered as Gaussian spots (see the Materials and Methods section). Spatial maps are rendered from 10000 successive frames. (c) Sample-to-sample variation of center wavelengths. Error bars are fwhm. (d) Temporal evolution of the number of localizations per frames (acquisition with $50 \mathrm{~ms}$ sampling and $20 \mathrm{~ms}$ exposure time). (e) Temporal evolution of the spectral distribution. The blue zone indicates the region used for reconstructing the spatial images in panel $\mathrm{b}$ and the distribution in panel a.

$\mathrm{hBN}$ materials as well as irradiation induced surface defects in bulk exfoliated hBN materials. Similar operational principles could be applied to transition-metal dichalcogenides at cryogenic temperatures $^{4-7}$ or other wide-band-gap materials at room temperature such as aluminum nitride, silicon carbide, and perovskites. $^{21,22}$ Our approach opens up broad perspectives in the use of SMLM techniques for wide-field characterization of defects in a variety of materials and paves the way for the wide-field quantitative multidimensional imaging of optically active defects. ${ }^{23}$

Experimental Setup. Our setup is based on a wide-field spectral super-resolution imaging scheme, using a prism in the detection path, previously developed in a biological context, ${ }^{24-26}$ and with recent extension in physical science fields such as chemistry. ${ }^{27}$ As shown schematically in Figure la,b, we excite the hBN samples using a $561 \mathrm{~nm}$ laser. The energy of the excitation laser $(2.21 \mathrm{eV})$ is well below the band gap of the $\mathrm{hBN}$ material $(\sim 6 \mathrm{eV})$, leading to the selective excitation of defects with energies well within the band gap of the material. The excitation beam from the laser is focused onto the back focal plane of a high-numerical-aperture oil-immersion microscope objective, leading to the wide-field illumination of the sample. hBN samples are deposited on silicon chips and immersed in water to prevent changes of refractive index in the optical path. Wide-field photoluminescence emission from the sample is collected by the same objective and separated from the excitation laser using dichroic and emission filters. The emission light is then split through two paths using a beam splitter (Figure 1b). Part of the emission going through Path 1 is directly projected onto one-half of an electron multiplying charge-coupled device (EMCCD) chip with a back-projected pixel size of $100 \mathrm{~nm}$ (Figure 1d, path 1). Emission from individual defects leads to the appearance of diffraction-limited spots on the camera chip (Figure 1d, red boxes), which can then be localized with subnanometer accuracy, with a localization uncertainty, $\sigma_{x, y} \approx \frac{\sigma_{\mathrm{PSF}}}{\sqrt{N}}$, where $\sigma_{\mathrm{PSF}} \approx 150 \mathrm{~nm}$ is the standard deviation of the Gaussian fit of emitter's intensity (corresponding to a diffraction-limited spot fixed by the Point Spread Function with a full width at half-maximum, fwhm, of $\sim 350 \mathrm{~nm}$ ), and $N$ is the number of photons emitted by the defect during the acquisition of one frame. The second path consists of an equilateral calcium fluoride $\left(\mathrm{CaF}_{2}\right)$ prism. This dispersive element leads to an approximately linear shift $\Delta y_{\text {SPEC }}$ (pixels) in the photoluminescence signal of each emitters relative to their emission wavelength $\lambda$, such that $\Delta y_{\mathrm{SPEC}} \approx a \times \lambda$, with $a \approx 0.25 \mathrm{px} / \mathrm{nm}$ (see Figure S1). As shown in Figure 1e, the one-to-one correspondence between the simultaneous images of emitters in the spatial and spectral channels allows the mapping of the spectrum of each individual emitter. The sample is further mounted on a piezoelectric scanner, and vertical drift is compensated using an IR-based feedback loop. ${ }^{28}$ Residual lateral sub-pixel drift is compensated through post-processing using cross-correlations between reconstructed super-resolved images (see the Methods and Materials section for further experimental and computational details).

Results and Discussion. Continuous green laser illumination of the flakes leads to the photoswitching (blinking) of the emitters between bright and dark states. ${ }^{18}$ As shown in 

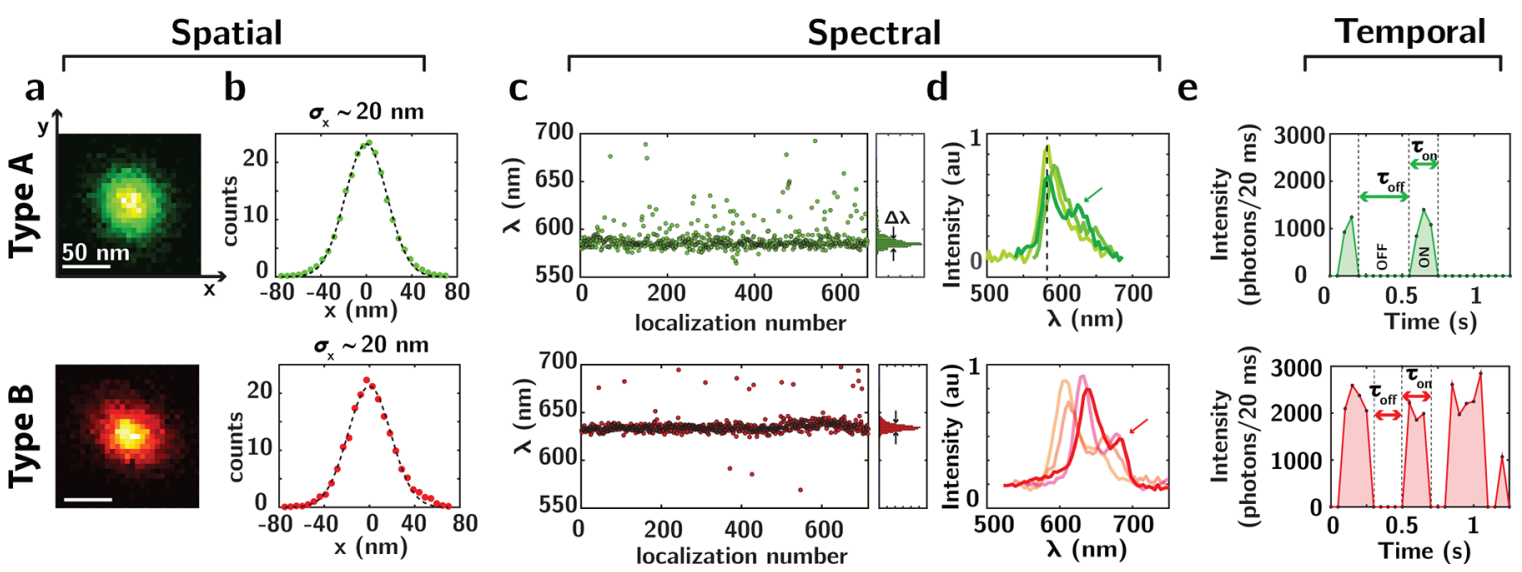

Figure 3. Spatial, spectral, and temporal dynamics for the two types of defects. (a) Super-resolved image of individual emitters, rendered as spatial (2D) histogram of localization coordinates with a $5 \mathrm{~nm}$ pixel size. (b) Projected distribution along the $x$ axis along with Gaussian fit (dotted line), allowing the determination of localization uncertainty $\sigma_{x}$. (c) Variation of the mean emission wavelength over successive localizations and histogram of emission wavelength. (d) Representative spectra of emitters. The vertical dashed line represents mean emission wavelength. Green and red arrows show, respectively, phonon sidebands in type A and type B emitters. (e) Time trace of emission intensity, showing intermittency in the emission, leading to successive "on" and "off" states. All measurements presented here are acquired with $50 \mathrm{~ms}$ sampling and $20 \mathrm{~ms}$ exposure time.

Figure 1d, due to this blinking behavior, only a small subset of emitters is active between each frame. This sparse activation allows for the spatial localization of individual emitters between each camera frame, and subsequent reconstruction of a spatial and spectral map by summing up successive images, as presented in Figure 1e.

As shown in Figure 2, our wide-field spectral SMLM scheme allows us to map the spectral properties of emitters present in the hBN flakes. We first plot in Figure 2a the distribution of center emission spectrum for a CVD-grown flake. As can be seen in this distribution, two families of emitters stand out clearly and are characterized by emission spectra centered approximately around $\lambda_{1} \approx 585 \mathrm{~nm}$ ("green emitters") and $\lambda_{2}$ $\approx 640 \mathrm{~nm}$ ("red emitters"). We can further map the spatial position of each type of emitters in the reconstructed spatial maps of Figure $2 \mathrm{~b}$ by summing up individual localization events (as depicted schematically in Figure 1e). The brighter dots on the map thus represent the most active defects, which are emitting through most of the acquired frames. This spectral map allows for a direct estimation of the spatial localization of each defect type. Despite the presence of areas with varying densities of defects in each flake, no clear spatial segregation is observed between red and green emitters because they are homogeneously represented throughout the sample.

This type of multimodal spectral distribution is observed throughout our samples. We report in Figure $2 c$ the values of center wavelength for 5 distinct flakes, obtained using similar growth conditions and transfer procedures. Note that several modes can occasionally be observed for the red emitters (flakes 2 and 4 in Figure 2c; see Figure S2 for details). Overall, emitters centered around $585 \mathrm{~nm}$ have relatively narrow spectral line width (fwhm of $\sim 15 \mathrm{~nm}$ ), consistent with recent report on similar CVD-grown materials, ${ }^{29}$ while the second group of emitters (with wavelength between approximately 610 and $650 \mathrm{~nm}$ ) has a larger spectral line width and show a relatively large sample-to-sample variation, which can be attributed to a variation in the local mechanical ${ }^{30,31}$ and electrostatic ${ }^{29}$ environment associated with each flake (e.g., due to residual strains developed during the transfer process). Finally, we show in Figure 2d,e the evolution of the number of localizations per frames and the spectral distribution over more than $30 \mathrm{~min}$. As is depicted in Figure 2d,e, the number of localizations per frame decreases exponentially, with a characteristic time $\tau \approx 10 \mathrm{~s}$ due to bleaching of the emitters, while spectral emission remains stable, with progressive reduction of the fwhm of red emitters probably due to bleaching. Importantly, localizations and wavelength matching can be performed as fast as 20 per frame, showing the potential of the technique for large-area and high-throughput mapping in dense samples. Bleaching, in turn, can happen due to the irreversible photo-oxidation of defects, exposed to the external environment due to the $2 \mathrm{D}$ nature of the flakes.

We now proceed in Figure 3 to the characterization of the spatial, spectral, and temporal dynamics of individual emitters in $\mathrm{hBN}$ using our wide-field SMLM spectral and spatial superresolution scheme. We show that at least two types of defects are responsible for the observed emission lines in CVD-grown flakes (Figure 2a-c). In Figure 3a, we first report superresolved images of individual emitters, rendered as spatial histograms of localizations using a pixel size of $5 \mathrm{~nm}$. As shown in Figure 3b, we obtain approximately Gaussian distributions, corresponding to spatial uncertainty $\sigma_{x}$ between 15 and $20 \mathrm{~nm}$. This value is slightly larger than the estimated spatial uncertainty of each localization based on photon counts (of the order of $7 \mathrm{~nm}$ ), which is probably due to residual drift. Remarkably, over the entire number $N \approx 1000$ of localizations we obtain a corresponding final uncertainty for the individual defect center position as low as $\frac{\sigma_{x}}{\sqrt{N}}<1 \mathrm{~nm}$ for the most active defects. The central emission wavelength of individual defects (Figure 3c) and full emission spectrum (Figure 3d) can then be measured using the procedure described in Figure 1d. As shown in Figure 3c, for the 2 representative types of emitters, relatively constant spectral emission is observed over 600 successive localization frames, with distribution width $\Delta \lambda \approx 5$ nm.

Representative spectra for the first defect type ("type A") are shown in green in Figure $3 \mathrm{~d}$. These defects have a mean emission wavelength centered along the first peak $\lambda_{1} \approx 585 \mathrm{~nm}$ in the spectral histogram of Figure 2a,c. Emission spectrum is asymmetric, and a phonon sideband can be occasionally 
resolved on some spectra (Figure 3d, green arrow). The second type of defect ("type B") is characterized by a mean emission wavelength centered along the second peak $\lambda_{2} \approx$ $610-650 \mathrm{~nm}$ of the spectral histogram. The spectrum shows a clear Zero Phonon Line and Phonon Sideband, leading to an energy detuning of $\sim 140 \mathrm{meV}$, consistent with previously published work. ${ }^{32-34}$ Remarkably, this phonon sideband is also visible on the ensemble histograms, in the form of a third local maximum in the spectral distribution (Figure 2a, red arrow). A representative time trace for the emission of each individual emitter is shown in Figure 3e. All defects systematically exhibit a blinking behavior, characterized by emission intermittency and successive "on" and "off" events.

To verify the generality of our approach, we characterized defects in a second class of hBN materials. We started from high-quality bulk hBN crystals, ${ }^{35}$ which were exfoliated and deposited on $\mathrm{Si} / \mathrm{SiO}_{2}$ substrate (see Figure S3). Few emitters, corresponding to both type A and type $\mathrm{B}$, were observed on the as-exfoliated flakes, reflecting the high quality of the bulk hBN material (see Figure S4). To deterministically induce defects in the structure, we exposed the exfoliated crystals to $30 \mathrm{~s}$ of oxygen plasma treatment ${ }^{36}$ (see the Materials and Methods section). As shown in Figure $4 a$, this leads to the
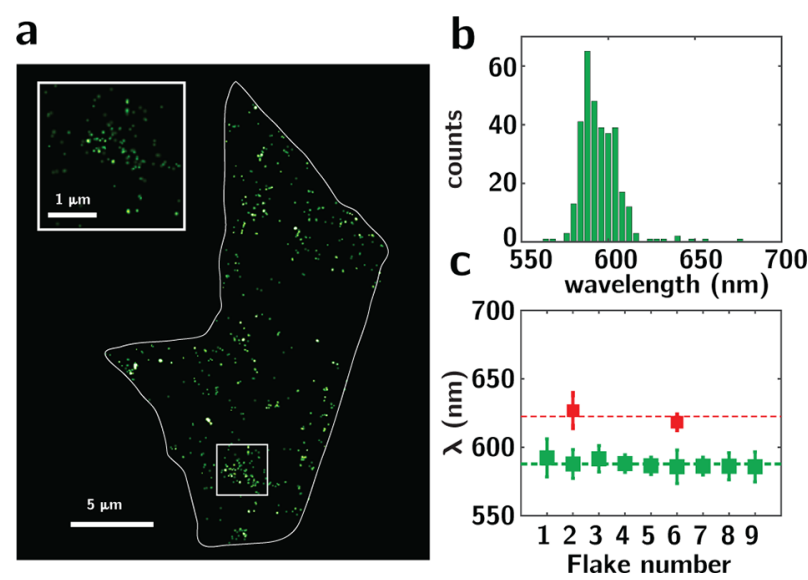

Figure 4. Spatial and spectral characterization of plasma-treated exfoliated hBN flakes. (a) Reconstructed map of individual emitters in exfoliated hBN flake, submitted to oxygen plasma for $30 \mathrm{~s}$ (see the Materials and Methods section). (b) Distribution of emission wavelength. (c) Sample-to-sample variation of the emission wavelength. Bin size is $4 \mathrm{~nm}$.

creation of emitters at the surface of the flakes with similar blinking behavior as in CVD-grown materials, allowing straightforward localization using SMLM-based spectral super-resolution mapping (representative time trace shown in Figure S5). Interestingly, as illustrated in Figure $4 b, c$, the spectral distribution in most of the investigated samples is characterized by a single emission wavelength, showing preferential creation of type A emitters by plasma treatment. Surprisingly, some of the investigated flakes also showed a significant population of type B emitters (Figure 4c flakes number 2 and 6 and Figure S6), although most of these emitters are unstable and bleach irreversibly after a few tens of seconds. This differential bleaching could be attributed to distinct chemical reactivity of the two populations of defects.

Focusing on CVD-grown hBN, we now turn in Figure 5 to the photophysical properties of emitters and specifically their intensity distribution and blinking kinetics. We show in Figure
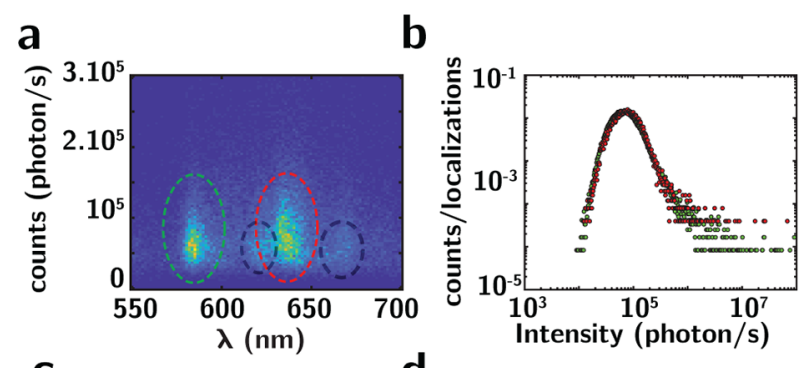

C

d
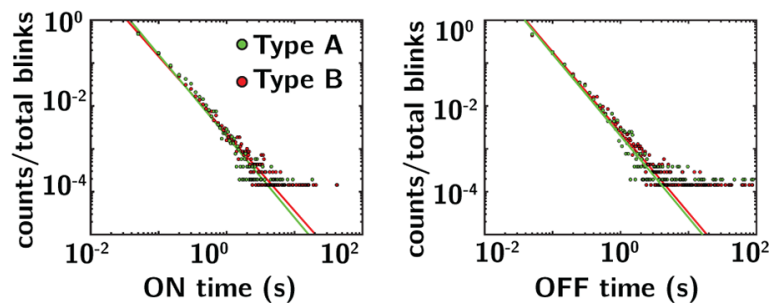

Figure 5. Photophysical properties of emitters in CVD hBN. (a) 2D histogram of photon counts as a function of emission wavelength. The two main clustered distributions are circled in green and red. The phonon sidebands of type A and type B emitters are circled in black. (b) Histogram of emission intensity. Red and green dots correspond to each circled distribution in panel a. The normalized histograms of (c) on and (d) off times for type A and type B emitters Materials and Methods. Straight lines indicate the power-law scaling of each distribution.

5a a $2 \mathrm{D}$ histogram of photon counts as a function of the emission wavelength for the flake investigated in Figure 2. A pair of clusters (green and red dashed circles) corresponding to type $A$ and type $B$ defects identified in Figures $2 a$ and 3 stand out clearly, with a high brightness of the order of $\sim 5 \times 10^{4}$ photons per second. As evidenced on the map, no clear difference of brightness can be made between each type of defects. The phonon sideband for type A and type B emitters can be identified in this histogram (black circled clusters). To obtain more insight into the properties of the defects in terms of photon emission, we show in Figure $5 b$ the full distribution of emission intensity by grouping the defects according to their wavelength. Interestingly, we observe a long tail in the intensity distribution, in strong contrast to the Poissonian distribution classically expected for non-blinking emitters. ${ }^{37}$

Another interesting feature of the emitters investigated here is their blinking kinetics, also observed in several other studies ${ }^{18,34,37}$ but contrasting with other reports of long-term emission stability for the emitters in $\mathrm{hBN} .{ }^{10,36}$ To gather more insight into this blinking behavior, we plot in Figure $5 \mathrm{c}, \mathrm{d}$ the distributions of "on" and "off" time for defect types A and B (see the Materials and Methods section). We observe clear power-law distributions, with $P_{\text {on/off }}(t) \approx t^{\alpha_{\text {on } / \text { off }}}$, and $\alpha_{\text {on }} \approx \alpha_{\text {off }} \approx 1.9 \pm 0.2$ (red and green lines, Figure $5 \mathrm{c}, \mathrm{d}$ ). Remarkably, these power-law distributions and the associated exponents are consistent with previously observed blinking kinetics of quantum dots, ${ }^{38,39}$ where blinking is attributed to ionization of the quantum dots and escape of photoexcited carriers toward surrounding charge traps. Importantly, the power-law scaling characterizes the absence of intrinsic timescales in the blinking behavior and can be interpreted as being due to the distribution of charge traps at the surface of the material. Similar mechanisms due to the photoinduced ionization and change of the charge state of the defects were 
proposed to explain blinking in NV centers in diamond. ${ }^{40}$ The blinking behavior observed in our samples might thus take its origin from charge separation or ionization of the defects, as the monolayer nature of the CVD-grown hBN flakes, and the creation of surface defects in plasma-treated hBN crystals, might allow, in both cases, for a facilitated escape of photoexcited charge carriers toward surrounding charge traps. This observation suggests a rationale behind the increased photostability observed in annealed samples as a way to desorb impurities acting as charge traps. Remarkably, the observed similarity in blinking kinetics suggests similar charge affinities for each types of defects (red and green dots, Figure $5 c, d)$. Finally, the long tail in the distribution of emission intensity, shown in Figure $5 b$, could be related to the observed power-law distribution of blinking times.

An important question remains to understand the reasons for the spectral heterogeneity evidenced in our study and the presence of different emission lines and defects states in CVD grown $\mathrm{hBN}$ and irradiated bulk $\mathrm{hBN}$ crystals. In the quest toward the assignment of a precise chemical structure to optically active defects in hBN materials, recent works have shown a widely heterogeneous spectral response of defects in $\mathrm{hBN}{ }^{32,41}$ which was attributed to different chemical structures $^{42,43}$ as well as differences in the charge states, ${ }^{34,44}$ local dielectric, ${ }^{33}$ electrostatic $^{29}$ and strain environment ${ }^{30}$ as well as temperature. ${ }^{41}$ Multimodal emission lines with modes around 585 and $630 \mathrm{~nm}$ were also observed in several studies. $^{34,43}$ While the blinking behavior observed for each types of defects (Figure 3e) is probably due to reversible ionization or charge trapping, it is also concurrent with clear spectral stability for the emission in the "on" state (Figure 3c). Furthermore, the observation of similar blinking characteristics for types A and B defects (Figure 5c,d) suggests similar charge affinities for these two populations. The differences in the spectral shape and zero phonon line distribution of type A and $\mathrm{B}$ defects (Figures $2 \mathrm{c}$ and $3 \mathrm{~d}$ ) could thus point to distinct chemical structures for these two populations, variations in their local dielectric environment or distinct charge states. Furthermore, the large variation in spectral emission amonst type $\mathrm{B}$ defects within different CVD-grown flakes might be attributed to residual strain developed during the transfer process. Despite their relatively large uncertainty, we can expect theoretical predictions from density functional theory to further guide the identification of the exact chemical structure of each type of defects ${ }^{45}$ based on their emission wavelength and spectra. It is noteworthy that $\mathrm{Adbi}$ and co-workers ${ }^{46}$ reported a zero phonon line for $N_{\mathrm{B}} V_{\mathrm{N}}$ and $V_{\mathrm{B}}{ }^{-}$defects of 2.05 and $1.92 \mathrm{eV}$, respectively, corresponding to 605 and $647 \mathrm{~nm}$ emission wavelength, which is in relatively fair agreement with our experimental results. However, the fact that we find predominantly one defect type in plasma treated exfoliated flakes might also suggest that type A correspond to intrinsic defects, while type $\mathrm{B}$ might occur due to substitutional doping with various impurities, such as $C_{\mathrm{B}} V_{\mathrm{N}}{ }^{47}$ or to various oxygen or hydrogen complexes or interstitial defects. ${ }^{48}$ Critically, the ability to assign defined chemical structures to optically active defects in hBN will only be possible through studies of the statistical properties of the spectral response of defects submitted to various growth conditions and post-growth treatments.

Conclusions. We have shown the potential of spectral SMLM techniques for wide-field and high-throughput spectral mapping and characterization of defects in hBN materials. Our methodology allows the determination of statistical ensemble spectral properties as well as the extraction of spatial, spectral, and temporal dynamics of single defects. We identify at least two types of defects in CVD grown materials, while irradiated exfoliated flakes show predominantly one type of defect. Further analysis of the blinking kinetics of optical emitters suggests that blinking is due to transient trapping of photoexcited charge carriers at the material's surface, similar to what is observed in quantum dots. This behavior provides strategies for blinking reduction and increase of photostability through encapsulation of defects and reduction of charge traps via thermal annealing. Our study demonstrates the potential of spectral SMLM as a wide-field and high-throughput characterization technique in material science and paves the way toward multidimensional mapping of defects' properties.

Materials and Methods. Optical Setup. The sample is excited using a $561 \mathrm{~nm}$ laser (Monolitic Laser Combiner 400B, Agilent Technologies). Excitation power during imaging, measured at the back focal plane of the microscope objective varies from 20 to $65 \mathrm{~mW}$, corresponding to power densities ranging from 250 to $800 \mathrm{~kW} / \mathrm{m}^{2}$. We used a high-numericalaperture oil-immersion microscope objective (Olympus TIRFM 100×, NA: 1.45). Wide-field photoluminescence emission from the sample is collected by the same objective and separated from the excitation laser using dichroic and emission filters (ZT488/561rpc-UF1 and ZET488/561m, Chroma). Paths 1 and 2 consist of two telescopes, ${ }^{26}$ sharing the same lens $(\mathrm{L} 1)$, with a respective magnification factor 1.6 (path 1, telescope L1-L2) and 1.4 (path 2, telescope L1-L3). Lenses are achromatic doublet lenses (Qioptic, L1: f/100, L2: $\mathrm{f} / 160, \mathrm{L3}: \mathrm{f} / 140$ ). The prism (PS863, Thorlabs) is placed at the Fourier plane between L1 and L3, at the angle of minimum deviation. The sample is mounted on a piezoelectric scanner (Nano-Drive, MadCityLabs) to compensate for vertical drift using an IR-based feedback loop. ${ }^{28}$ An EMCCD camera (Andor iXon Life 897) is used with an EM gain of 150 .

SMLM . Localizations. Emitters in the spatial and spectral channels are localized using the imageJ plugin Thunderstorm. ${ }^{49}$ Briefly, a wavelet filter is applied to each frame. Peaks are then fitted by 2D-integrated Gaussians. In the spatial channel, only emitters with intensity at least twice the standard deviation of the first wavelet level are considered.

Spectral Calibration. Calibration between spatial and spectral channels is conducted using red fluorescent beads (20 nm FluoSpheres carboxylated-modified, 580/605). First, we calibrate the field-of-view transformation between spatial and spectral channels. For an emitter with a given wavelength, the relation between its position in the spatial and spectral channel is well approximated by a relation of the form ( $x_{\mathrm{SPEC}}$, $\left.y_{\mathrm{SPEC}}\right)=A\left(x_{\mathrm{LOC}}, y_{\mathrm{LOC}}\right)+B$, where $\left(x_{\mathrm{SPEC}}, y_{\mathrm{SPEC}}\right)$ and $\left(x_{\mathrm{LOC}}\right.$, $\left.y_{\text {LOC }}\right)$ correspond to the position vector of the emitter in the spectral and spatial channels, respectively; $A$ is a $2 \times 2$ matrix; and $B$ is a vector. Coefficients for $A$ and $B$ are calibrated by raster scanning a fiducial marker of controlled emission wavelength and mapping its position in both channels. In a second step, band-pass filters with a central wavelength of 600 , 630 and $650 \mathrm{~nm}$, respectively, and a fwhm of $10 \mathrm{~nm}$ (Thorlabs, FB600-10) are used to calibrate the relation between the emission spectrum and the vertical shift in the spectral channel. The relation between the vertical shift $\Delta y_{\text {SPEC }}$ (pixels) of the emission spectra and the emission wavelength $\lambda$ is approxi- 
mated well by a linear relation, such that $\Delta y_{\text {SPEC }} \approx a \times \lambda$, with $a \approx 0.25 \mathrm{px} / \mathrm{nm}$ (see Figure S1).

Spectral Assignment. To assign a spectra to an emitter localized in the spatial channel at $\left(x_{\mathrm{LOC}}, y_{\mathrm{LOC}}\right)$, we compute its projected position $\left(x_{\mathrm{SPEC}}\left(\lambda_{0}\right), y_{\mathrm{SPEC}}\left(\lambda_{0}\right)\right)$ in the spectral channel for a fixed emission wavelength of $\lambda_{0}=650 \mathrm{~nm}$. A pair-search algorithm finds the closest localizations ( $x_{\text {SPEC }}^{\prime}$, $y_{\text {SPEC }}^{\prime}$ ) in a vertically elongated rectangular zone around $\left(x_{\mathrm{SPEC}}\left(\lambda_{0}\right), y_{\mathrm{SPEC}}\left(\lambda_{0}\right)\right)$. The corresponding central emission wavelength $\lambda$ is then estimated as $\lambda=\lambda_{0}+1 / a\left(y_{\text {SPEC }}\left(\lambda_{0}\right)-\right.$

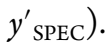

Spectra Generation. Measured spectra in Figure $3 \mathrm{~d}$ are obtained by averaging the spectrum of single emitters over all frames for which spectral assignment is successful.

Image Generation. SMLM images (Figures $2 \mathrm{~b}$ and $4 \mathrm{a}$ ) are generated as probability maps by plotting 2D Gaussian centered on each position with a standard deviation equal to $20 \mathrm{~nm}$.

Drift Correction. Lateral drift is corrected using crosscorrelation between reconstructed super-resolved images on CVD-grown materials. No lateral drift correction is applied on exfoliated hBN flakes because the lower density of defects leads to weak cross-correlations between reconstructed images.

Blinking Kinetics. We obtain the blinking kinetics of type A and type B defects (Figure 5c,d) by following the time-trace of 9 type A defects and 14 type B defects (histogram of mean emission wavelength shown in Figure S7). The "off" state is clearly defined by the absence of localization (Figure $3 \mathrm{e}$ ).

Sample Preparation. CVD-Grown Materials. CVD-grown materials are produced under similar conditions, as described elsewhere $^{50}$ (see Figure S8 for SEM images of flakes on Fe foil). Briefly, as-received Fe foil (100 $\mu \mathrm{m}$ thick, Goodfellow, $99.8 \%$ purity) is loaded in a customized CVD reactor (base pressure $1 \times 10^{-6} \mathrm{mbar}$ ) and heated to $\sim 940{ }^{\circ} \mathrm{C}$ in $\mathrm{Ar}$ (4 mbar), followed by annealing in $\mathrm{NH}_{3}$ (4 mbar). For the growth, $1 \times 10^{-2}$ mbar $\mathrm{NH}_{3}$ is used as a carrier gas, and $6 \times$ $10^{-4} \mathrm{mbar}$ borazine $(\mathrm{HBNH})_{3}$ is introduced into the chamber for $30 \mathrm{~min}$. The growth is quenched by turning off the heater, allowing a cooling rate of about $200{ }^{\circ} \mathrm{C} / \mathrm{min}$.

The h-BN domains are transferred onto $\mathrm{SiN}_{x}$ chips using the electrochemical bubbling method ${ }^{51}$ with PMMA as support layer. After transfer, PMMA is removed by successive $1 \mathrm{~h}$ rinses in hot acetone ( 3 rinses), hot IPA ( 1 rinse), and hot DI water (1 rinse). Remaining PMMA contamination is further removed through overnight annealing at $400{ }^{\circ} \mathrm{C}$ in an argon atmosphere.

Exfoliated Flakes. hBN multilayer flakes are exfoliated from high-quality bulk crystals ${ }^{35}$ and deposited onto $\mathrm{SiO}_{2}$ chips (Figure S3). Type A and type B defects are detected at low concentrations in just-exfoliated flakes (Figure S4). To deterministically create defects, hBN crystals are further exposed to a $30 \mathrm{~s}$ oxygen plasma at $100 \mathrm{~mW}$ under $30 \mathrm{sccm}$ $\mathrm{O}_{2}$ flow. No annealing was performed.

Sample Imaging. Chips with deposited hBN flakes and CVD-grown materials are placed upside down on round coverslips (no. 1.5 Micro Coverglass, Electron Microscopy Sciences, $25 \mathrm{~mm}$ in diameter) previously cleaned in oxygen plasma for $5 \mathrm{~min}$. Imaging is further performed in water at room temperature to improve the optical contrast and prevent a discontinuous change in the refractive index. Performing experiments in air by transferring CVD-grown $\mathrm{hBN}$ flakes directly on a glass coverslip, we observed a similar spectral signature as that shown in Figure 2, showing that water has little effect on the observed spectral response of this material.

\section{ASSOCIATED CONTENT}

\section{S Supporting Information}

The Supporting Information is available free of charge on the ACS Publications website at DOI: 10.1021/acs.nanolett.9b00178.

Figures showing the calibration of spectral SMLM, the distributions of central emission wavelengths for CVDgrown and exfoliated $\mathrm{hBN}$, representative time trace, and wide-field and SEM images (PDF)

\section{AUTHOR INFORMATION}

\section{Corresponding Authors}

*E-mail: jean.comtet@gmail.com.

*E-mail: jiandong.feng@zju.edu.cn.

*E-mail: aleksandra.radenovic@epfl.ch.

ORCID $\odot$

Stephan Hofmann: 0000-0001-6375-1459

Aleksandra Radenovic: 0000-0001-8194-2785

\section{Author Contributions}

A.R. and J.F. conceived of the project and designed experiments. J.C. developed the experimental setup for spectral imaging and performed the experiments with help from E.G. and N.V. V.B. and S.H. produced the CVD-grown hBN samples. K.W. and T.T. produced the bulk hBN crystals. J.C. wrote the paper with input from all authors. A.R. supervised the project.

\section{Funding}

This work was financially supported by the Swiss National Science Foundation (SNSF) Consolidator grant (BIONIC BSCGI0_157802) and CCMX project ("Large Area Growth of 2D Materials for device integration"). K.W. and T.T. acknowledge support from the Elemental Strategy Initiative conducted by the MEXT, Japan and the CREST (JPMJCR15F3), JST. V.B. and S.H. acknowledge funding from the European Union's Horizon 2020 research and innovation program under grant agreement no. 785219.

\section{Notes}

The authors declare no competing financial interest.

\section{ACKNOWLEDGMENTS}

We thank Jean-Baptiste Sibarita and Corey Butler for initial help and experiments on spectral SMLM. We also acknowledge valuable discussions with Adrien Descloux and Kristin Grussmayer. We thank Ahmet Avsar for his help with the transfer of exfoliated hBN, Michael Graf for fabricating the marked silicon substrates, and Ivor Lončaric for the discussion on the interpretation of our results.

\section{REFERENCES}

(1) Mizuochi, N.; Makino, T.; Kato, H.; Takeuchi, D.; Ogura, M.; Okushi, H.; Nothaft, M.; Neumann, P.; Gali, A.; Jelezko, F.; et al. Nat. Photonics 2012, 6 (5), 299-303.

(2) Hong, S.; Grinolds, M. S.; Pham, L. M.; Le Sage, D.; Luan, L.; Walsworth, R. L.; Yacoby, A. MRS Bull. 2013, 38 (2), 155-161.

(3) Schirhagl, R.; Chang, K.; Loretz, M.; Degen, C. L. Annu. Rev. Phys. Chem. 2014, 65 (1), 83-105.

(4) Koperski, M.; Nogajewski, K.; Arora, A.; Cherkez, V.; Mallet, P.; Veuillen, J. Y.; Marcus, J.; Kossacki, P.; Potemski, M. Nat. Nanotechnol. 2015, 10 (6), 503-506. 
(5) He, Y. M.; Clark, G.; Schaibley, J. R.; He, Y.; Chen, M. C.; Wei, Y. J.; Ding, X.; Zhang, Q.; Yao, W.; Xu, X. Nat. Nanotechnol. 2015, 10 (6), 497-502.

(6) Srivastava, A.; Sidler, M.; Allain, A. V.; Lembke, D. S.; Kis, A.; Imamoglu, A. Nat. Nanotechnol. 2015, 10 (6), 491-496.

(7) Chakraborty, C.; Kinnischtzke, L.; Goodfellow, K. M.; Beams, R.; Vamivakas, A. N. Nat. Nanotechnol. 2015, 10 (6), 507-511.

(8) Tran, T. T.; Bray, K.; Ford, M. J.; Toth, M.; Aharonovich, I. Nat. Nanotechnol. 2016, 11 (1), 37-41.

(9) Mak, K. F.; Shan, J. Nat. Photonics 2016, 10 (4), 216-226.

(10) Aharonovich, I.; Englund, D.; Toth, M. Nat. Photonics 2016, 10 (10), 631-641.

(11) Nikolay, N.; Mendelson, N.; Sadzak, N.; Böhm, F.; Tran, T. T.; Sontheimer, B.; Aharonovich, I.; Benson, O. 2018, arXiv: 1812.02530. arXiv.org e-Print archive. https://arxiv.org/abs/1812.02530 (accessed on Dec 6, 2018).

(12) Branny, A.; Kumar, S.; Proux, R.; Gerardot, B. D. Nat. Commun. 2017, 8, 15053.

(13) Palacios-Berraquero, C.; Kara, D. M.; Montblanch, A. R. P.; Barbone, M.; Latawiec, P.; Yoon, D.; Ott, A. K.; Loncar, M.; Ferrari, A. C.; Atatüre, M. Nat. Commun. 2017, 8, 15093.

(14) Proscia, N. V.; Shotan, Z.; Jayakumar, H.; Reddy, P.; Dollar, M.; Alkauskas, A.; Doherty, M.; Meriles, C. A.; Menon, V. M.; Cohen, C. Optica 2018, 5 (9), 1128-1134.

(15) Dean, C. R.; Young, A. F.; Meric, I.; Lee, C.; Wang, L.; Sorgenfrei, S.; Watanabe, K.; Taniguchi, T.; Kim, P.; Shepard, K. L.; et al. Nat. Nanotechnol. 2010, 5 (10), 722-726.

(16) Ranjan, A.; Raghavan, N.; O’Shea, S. J.; Mei, S.; Bosman, M.; Shubhakar, K.; Pey, K. L. IEEE Int. Reliab. Phys. Symp. Proc. 2018, 4A11-4A16.

(17) Li, L. H.; Chen, Y. Adv. Funct. Mater. 2016, 26 (16), 25942608.

(18) Feng, J.; Deschout, H.; Caneva, S.; Hofmann, S.; Lončarić, I.; Lazić, P.; Radenovic, A. Nano Lett. 2018, 18 (3), 1739-1744.

(19) Wong, D.; Velasco, J.; Ju, L.; Lee, J.; Kahn, S.; Tsai, H. Z.; Germany, C.; Taniguchi, T.; Watanabe, K.; Zettl, A.; et al. Nat. Nanotechnol. 2015, 10 (11), 949-953.

(20) Fei, Z.; Rodin, A. S.; Gannett, W.; Dai, S.; Regan, W.; Wagner, M.; Liu, M. K.; McLeod, A. S.; Dominguez, G.; Thiemens, M.; et al. Nat. Nanotechnol. 2013, 8 (11), 821-825.

(21) Merdasa, A.; Tian, Y.; Camacho, R.; Dobrovolsky, A.; Debroye, E.; Unger, E. L.; Hofkens, J.; Sundström, V.; Scheblykin, I. G. ACS Nano 2017, 11 (6), 5391-5404.

(22) Ponseca, C. S.; Pullerits, T.; Yartsev, A.; Sundstro, V.; Scheblykin, I. G. Nano Lett. 2015, 15 (3), 1603-1608.

(23) Nicovich, P. R.; Owen, D. M.; Gaus, K. Nat. Protoc. 2017, 12 (3), 453

(24) Moon, S.; Yan, R.; Kenny, S. J.; Shyu, Y.; Xiang, L.; Li, W.; Xu, K. J. Am. Chem. Soc. 2017, 139 (32), 10944-10947.

(25) Zhang, Z.; Kenny, S. J.; Hauser, M.; Li, W.; Xu, K. Nat. Methods 2015, 12 (10), 935-938.

(26) Yan, R.; Moon, S.; Kenny, S. J.; Xu, K. Acc. Chem. Res. 2018, 51 (3), 697-705.

(27) Kim, D.; Zhang, Z.; Xu, K. J. Am. Chem. Soc. 2017, 139 (28), 9447-9450.

(28) Deschout, H.; Lukes, T.; Sharipov, A.; Szlag, D.; Feletti, L.; Vandenberg, W.; Dedecker, P.; Hofkens, J.; Leutenegger, M.; Lasser, T.; et al. Nat. Commun. 2016, 7, 13693.

(29) Mendelson, N.; Xu, Z.-Q.; Tran, T. T.; Kianinia, M.; Scott, J.; Bradac, C.; Aharonovich, I.; Toth, M. ACS Nano 2019. DOI: $10.1021 /$ acsnano.8b08511

(30) Grosso, G.; Moon, H.; Lienhard, B.; Ali, S.; Efetov, D. K.; Furchi, M. M.; Jarillo-Herrero, P.; Ford, M. J.; Aharonovich, I.; Englund, D. Nat. Commun. 2017, 8 (1), 705.

(31) Xue, Y.; Wang, H.; Tan, Q.; Zhang, J.; Yu, T.; Ding, K.; Jiang, D.; Dou, X.; Shi, J. J.; Sun, B. Q. ACS Nano 2018, 12 (7), 7127-7133.

(32) Choi, S.; Tran, T. T.; Elbadawi, C.; Lobo, C.; Wang, X.; Juodkazis, S.; Seniutinas, G.; Toth, M.; Aharonovich, I. ACS Appl. Mater. Interfaces 2016, 8 (43), 29642-29648.
(33) Tran, T. T.; Elbadawi, C.; Totonjian, D.; Lobo, C. J.; Grosso, G.; Moon, H.; Englund, D. R.; Ford, M. J.; Aharonovich, I.; Toth, M. ACS Nano 2016, 10 (8), 7331-7338.

(34) Martínez, L. J.; Pelini, T.; Waselowski, V.; Maze, J. R.; Gil, B.; Cassabois, G.; Jacques, V. Phys. Rev. B: Condens. Matter Mater. Phys. 2016, 94 (12), 1-5.

(35) Taniguchi, T.; Watanabe, K. J. Cryst. Growth 2007, 303 (2), $525-529$.

(36) Xu, Z. Q.; Elbadawi, C.; Tran, T. T.; Kianinia, M.; Li, X.; Liu, D.; Hoffman, T. B.; Nguyen, M.; Kim, S.; Edgar, J. H.; et al. Nanoscale 2018, 10 (17), 7957-7965.

(37) Tran, T. T.; Zachreson, C.; Berhane, A. M.; Bray, K.; Sandstrom, R. G.; Li, L. H.; Taniguchi, T.; Watanabe, K.; Aharonovich, I.; Toth, M. Phys. Rev. Appl. 2016, 5 (3), 463-466.

(38) Frantsuzov, P.; Kuno, M.; Jankó, B.; Marcus, R. A. Nat. Phys. 2008, 4 (7), 519-522.

(39) Efros, A. L.; Nesbitt, D. Nat. Nanotechnol. 2016, 11 (8), 661671

(40) Wrachtrup, J.; Neumann, P.; Jelezko, F.; Aslam, N.; Waldherr, G. New J. Phys. 2013, 15 (1), 013064.

(41) Jungwirth, N. R.; Calderon, B.; Ji, Y.; Spencer, M. G.; Flatté, M. E.; Fuchs, G. D. Nano Lett. 2016, 16 (10), 6052-6057.

(42) Chejanovsky, N.; Rezai, M.; Paolucci, F.; Kim, Y.; Rendler, T.; Rouabeh, W.; Fávaro De Oliveira, F.; Herlinger, P.; Denisenko, A.; Yang, S.; et al. Nano Lett. 2016, 16 (11), 7037-7045.

(43) Dietrich, A.; Bürk, M.; Steiger, E. S.; Antoniuk, L.; Tran, T. T.; Nguyen, M.; Aharonovich, I.; Jelezko, F.; Kubanek, A. Phys. Rev. B: Condens. Matter Mater. Phys. 2018, 98 (8), 2-6.

(44) Shotan, Z.; Jayakumar, H.; Considine, C. R.; Mackoit, M.; Fedder, H.; Wrachtrup, J.; Alkauskas, A.; Doherty, M. W.; Menon, V. M.; Meriles, C. A. ACS Photonics 2016, 3 (12), 2490-2496.

(45) Reimers, J. R.; Sajid, A.; Kobayashi, R.; Ford, M. J. J. Chem. Theory Comput. 2018, 14 (3), 1602-1613.

(46) Abdi, M.; Chou, J. P.; Gali, A.; Plenio, M. B. ACS Photonics 2018, 5 (5), 1967-1976.

(47) Tawfik, S. A.; Ali, S.; Fronzi, M.; Kianinia, M.; Tran, T. T.; Stampfl, C.; Aharonovich, I.; Toth, M.; Ford, M. J. Nanoscale 2017, 9 (36), 13575-13582.

(48) Weston, L.; Wickramaratne, D.; Mackoit, M.; Alkauskas, A.; Van De Walle, C. G. Phys. Rev. B: Condens. Matter Mater. Phys. 2018, 97 (21), 1-13.

(49) Ovesný, M.; Kř́žek, P.; Borkovec, J.; Švindrych, Z.; Hagen, G. M. Bioinformatics 2014, 30 (16), 2389-2390.

(50) Caneva, S.; Weatherup, R. S.; Bayer, B. C.; Blume, R.; CabreroVilatela, A.; Braeuninger-Weimer, P.; Martin, M. B.; Wang, R.; Baehtz, C.; Schloegl, R.; et al. Nano Lett. 2016, 16 (2), 1250-1261.

(51) Gao, L.; Ren, W.; Xu, H.; Jin, L.; Wang, Z.; Ma, T.; Ma, L. P.; Zhang, Z.; Fu, Q.; Peng, L. M.; et al. Nat. Commun. 2012, 3, 697699. 